\title{
Expression of MUC2 Gene Product in Mucinous Carcinoma of the Breast: Comparison with Invasive Ductal Carcinoma
}

\author{
Suguru Yonezawa', Mitsuharu Nomoto', Sumika Matsukita', Pei-Xiang Xing ${ }^{2}$, \\ Ian F. C. McKenzie ${ }^{2}$, John Hilkens ${ }^{3}$, Young S. Kim ${ }^{4}$ and Eiichi Sato ${ }^{1}$ \\ ${ }^{1}$ Department of Pathology, Kagoshima University School of Medicine, Kagoshima, 890, ${ }^{2}$ Austin Research Institute, \\ Austin Hospital, Heidelberg, Victoria, Australia, ${ }^{3}$ Division of Tumor Biology, The Netherlands Cancer Institute, \\ Amsterdam, The Netherlands and ${ }^{4}$ Gastrointestinal Research Laboratory, Veterans Administration Medical \\ Center, and University of California, San Francisco, U.S.A.
}

Received for publication March 15, 1995 and in revised form May 26, 1995

\begin{abstract}
We have previously reported that MUC2 gene product was dominantly expressed in the non-invasive carcinomas of the pancreas and liver (Cancer 71: 2191-2199, 1993; Int J Cancer 55: 82-91, 1993). Mucinous carcinoma (MC) of the breast usually shows less frequent lymph node metastasis and a more favorable outcome compared with invasive ductal carcinoma (IDC). In the present study, we examined the expression of MUC1 gene product (DF3, 139H2) and MUC2 gene product (anti-MRP, CCP58) immunohistochemically in 17 cases of MC (11 pure MCs, and 6 mixed MCs comprising MC and IDC) and 57 cases of IDC. Most of the pure MCs showed MUC1 positive (DF3: 11/11, 139H2: 11/11) and MUC2 positive (antiMRP: 10/11, CCP58: 11/11) pattern. Most of the mixed MCs showed MUC1 positive
\end{abstract}

(DF3: 6/6, 139H2: 6/6) and MUC2 positive (anti-MRP: 5/6, CCP58: 6/6) pattern in the MC areas, but MUC1 positive (DF3: 6/6, 139H2: 6/6) and MUC2 negative (anti-MRP: 0/6, CCP58: 0/6) pattern in the IDC areas. In contrast, most of the IDCs showed MUC1 positive (DF3: 54/57, 139H2: 53/57) and MUC2 negative (anti-MRP: 0/57, CCP58: 0/57) pattern, but never showed MUC1 positive and MUC2 positive pattern. Thus, the expression of MUC2 gene product is associated with the less aggressive biological properties of MC than IDC through the production of abundant extracellular mucin forming the characteristic configuration, since MUC2 glycoprotein is a secretory mucin, while MUC1 glycoprotein is membrane-associated.

Key words: Mucinous carcinoma, Invasive ductal carcinoma, Breast, MUC2 mucin, Immunohistochemistry

\section{Introduction}

Mucins are high molecular weight glycoproteins having oligosaccharides attached to serine or threonine residues of the apomucin protein backbone by $\mathrm{O}$ glycosidic linkages [11]. Synthesis and secretion of mucin is a common feature of glandular epithelial tissues, and alterations in the glycosylation of mucins have been described in cancer $[9-11,13,26,27,35]$. Besides the ex-

Supported in part by Grant-in-aid 06670196 from the Ministry of Education Science and Cultutre, Japan (S. Y.), and by USPHS Grant CA24321 from the National Cancer Institute, U.S.A. (Y.S. K.) and by the Veterans Affairs Medical Research Service, U.S.A. (Y.S. K.).

Correspondence to: Suguru Yonezawa, M. D., Second Department of Pathology, Kagoshima University School of Medicine, 8-35-1 Sakuragaoka, Kagoshima City, Kagoshima 890, Japan. tensive studies on carbohydrate chains of mucin antigens, the biochemical studies on the structures and the organ specificities of mucin core proteins also have been reported recently $[4,12,34]$. Our previous studies of the pancreatic and intrahepatic bile-duct tumors disclosed the dominant expression of MUC2 gene product in non-invasive carcinomas with a favorable prognosis [21, 32]. Namely, intraductal papillary tumors of the pancreas and bile-duct cystadenocarcinomas of the liver, both of which show good outcomes compared with invasive ductal carcinomas of the pancreas and cholangiocarcinomas, produce an abundant amount of mucin. Both of them usually express MUC2 gene product in the cytoplasm of the neoplastic cells. Mucinous carcinoma (MC) of the breast produces a large amount of extracellular mucinous substance, and shows less frequent lymph node metastasis and a more favorable outcome compared with invasive ductal car- 
cinoma (IDC) of the breast $[2,14,20,23]$. We hypothesized that MC of the breast also express MUC2 gene product. In the current study, we compared the expression of the mucin core protein antigens associated with MUC1 gene product (mammary-type apomucin) $[1,3,16,17,22$, 25, 30] and MUC2 gene product (intestinal-type apomucin) $[6,31]$ in MCs and IDCs using the immunohistochemical method.

\section{Materials and Methods}

\section{Tissue samples}

Surgical specimens from 17 cases of MC were obtained from the file of the Department of Pathology, Kagoshima University School of Medicine. The tumors with MC were divided into two types: "pure MC" (11 cases) consisting only of areas with small epithelial islands of solid tumor floating in abundant extracellular mucin [2, $14,20,23]$; and "mixed MC" (6 cases) in which IDC component was detected histologically besides the MC component $[2,14,20,23]$. Surgical specimens from 57 cases of IDC were obtained from the file of the Department of Pathology, Kagoshima Municipal Hospital. All the specimens were primary breast cancers from female patients undergoing mastectomy or excisional biopsy. All the specimens were fixed in formalin, embedded in paraffin and cut into $4 \mu \mathrm{m}$ serial sections for immunohistochemical staining, in addition to the usual hematoxylin and eosin staining.

\section{Antibodies}

Monoclonal antibody (MAb) DF3 (mouse IgG, ascites, Centocor CA15-3, Toray-Fuji Bionics, Lot No. 12445) $[1,15,24,25]$, and MAb 139H2 (mouse IgG, ascites) $[7,8]$ were used to identify the tandem repeat peptide of MUC1 gene product, although MAb DF3 binding to the mucin core protein may be enhanced by the presence of glycosidic linkages [25]. Polyclonal antibody (PAb) anti-MRP (purified rabbit IgG) [6] and MAb CCP58 (mouse IgG, ascites) [31] were used to identify the tandem repeat peptide of MUC2 gene product. Anti-MRP was produced using a synthetic MUC2 peptide containing the 23-amino acid threonine rich tandem repeat peptide of MUC2 apomucin, PTTTPISTTTMVTPTPTPTGTQT [6]. MAb
CCP58 was produced using a synthetic MUC2 peptide designated as MI-29 KYPTTTPISTTTMVTPTPTPTGTQTPTTT, and reacted with the MUC2-derived peptide but not with MUC1- or MUC3-derived peptides [31].

Biotinylated affinity-purified horse antimouse IgG and goat antirabbit IgG, and avidin-biotinylated horseradish peroxidase complex (ABC complex) were purchased from Vector Laboratories (Burlingame, CA) as the Vectastain Elite ABC Kit.

\section{Staining procedures}

The ABC immunoperoxidase method has been described previously $[21,32,35]$. Briefly, each section was deparaffinized, rehydrated and incubated at room temperature with $0.3 \%$ hydrogen peroxide in methanol for $30 \mathrm{~min}$ to block endogenous peroxidase and washed with phosphate-buffered saline (PBS, pH 7.4). Next, 2\% horse or goat serum in PBS was applied for $30 \mathrm{~min}$ at room temperature to prevent non-specific staining.

The sections for immunohistochemical study were incubated with primary antibodies [DF3, $1: 200 ; 139 \mathrm{H} 2$, 1:2000; anti-MRP, 1:600; CCP58, 1:1000 dilutions, respectively, in PBS with $1 \%$ bovine serum albumin (PBSBSA)] for $16 \mathrm{hr}$ at $4^{\circ} \mathrm{C}$. All the sections were washed three times with PBS and incubated with the biotinylated secondary antibodies, and washed three times with PBS. All the sections then were reacted with the $A B C$ complex for $30 \mathrm{~min}$. After washing with PBS three times, the sections were finally reacted with diaminobenzidine substrate for 10 to $30 \mathrm{~min}$, rinsed with tap water, stained with hematoxylin and mounted. Negative controls consisted of substituting PBS, nonimmune mouse or rabbit serum for the primary antibodies.

\section{Evaluation of the results by scoring}

The results of the antibody staining patterns were graded by the percentage of positively stained neoplastic cells as follows: - ; less than $5 \%$ of neoplastic cells stained; $+; 5-50 \%$ of neoplastic cells stained; and H; over $50 \%$ of neoplastic cells stained. We evaluated the stainings of cytoplasm, cell membrane and associated secretory products (luminal contents) separately, although the associated secretory products were not always present in IDCs, especially in the scirrhous type.

Table 1. Expression of MUCl and MUC2 gene products in mucinous carcinomas (MC) and invasive ductal carcinomas (IDC)

\begin{tabular}{|c|c|c|c|c|c|c|c|c|c|c|c|c|c|c|c|c|c|c|c|c|}
\hline & & & \multicolumn{8}{|c|}{ MUCl } & \multicolumn{10}{|c|}{ MUC2 } \\
\hline & \multicolumn{2}{|l|}{ Antibodies } & DF3 & c & m & $\mathrm{s}$ & $139 \mathrm{H} 2$ & c & $\mathrm{m}$ & $\mathbf{s}$ & anti & i-MRP & c & m & $\mathbf{s}$ & & CP58 & c & m & s \\
\hline \multirow{3}{*}{ MC } & Pure type $(n=11)$ & & $11(100 \%)$ & 6 & 11 & 10 & $11(100 \%)$ & 6 & 11 & 3 & 10( & (91\%) & 10 & 0 & 0 & & $100 \%)$ & 11 & 0 & 0 \\
\hline & \multirow{2}{*}{ Mixed type $(n=6)$} & MC aera & $6(100 \%)$ & 6 & 6 & 2 & $6(100 \%)$ & 6 & 6 & 1 & & $(83 \%)$ & 5 & 0 & 0 & \multicolumn{2}{|c|}{$6(100 \%)$} & 6 & 0 & 0 \\
\hline & & IDC area & $6(100 \%)$ & 6 & 6 & 4 & $6(100 \%)$ & 6 & 6 & 3 & 0 & $(0 \%)$ & 0 & 0 & 0 & 0 & $(0 \%)$ & 0 & 0 & 0 \\
\hline IDC & $(n=57)$ & & $54(95 \%)$ & 45 & 49 & 32 & $53(93 \%)$ & 41 & 48 & 13 & 0 & $(0 \%)$ & 0 & 0 & 0 & $\mathbf{0}$ & $(0 \%)$ & 0 & 0 & 0 \\
\hline
\end{tabular}




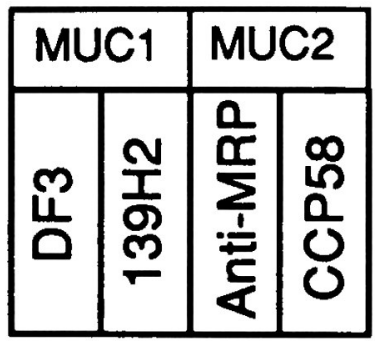

\section{Pure MC}

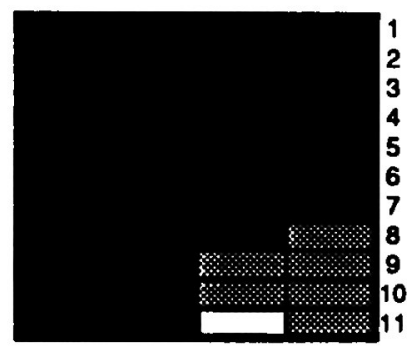

\section{Mixed MC}
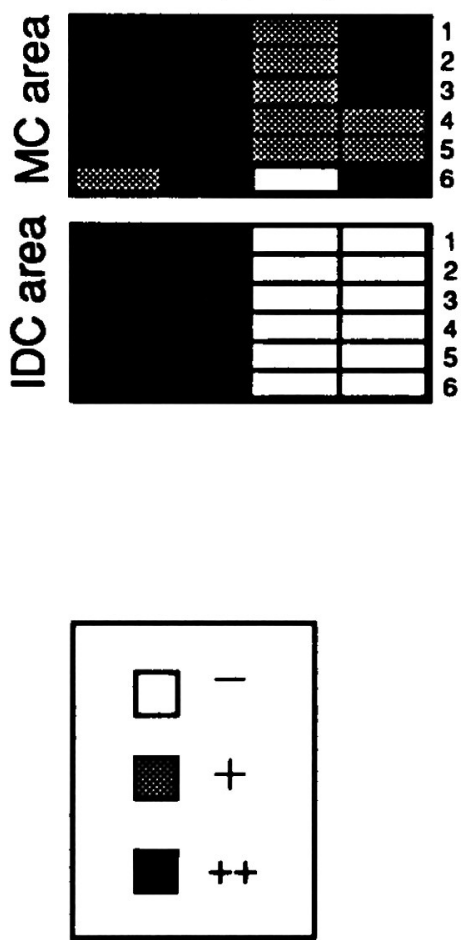
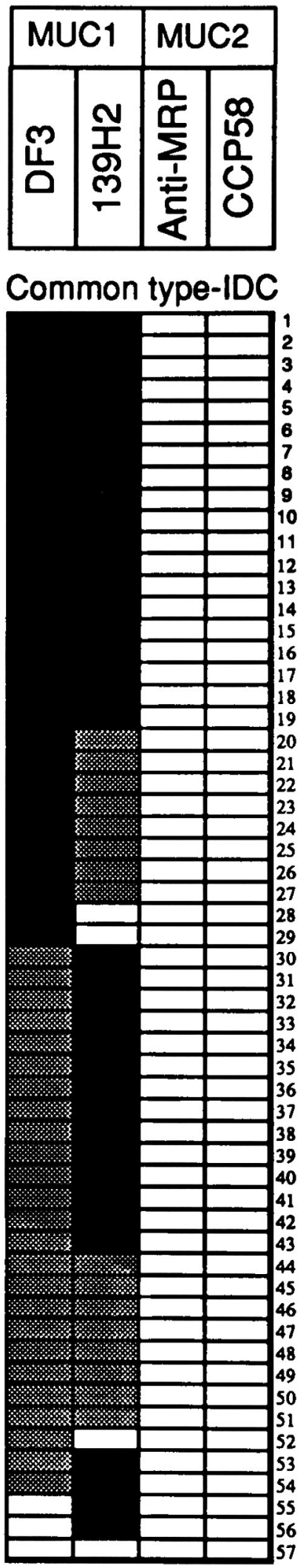

Fig. 1. Expression patterns and staining densities of MUC1 gene product and MUC2 gene product in each case studied. The highest positivity percentages in the stainings of cytoplasm, cell membrane and associated secretory products were scored as the representative grades ( - ; less than $5 \%$ of neoplastic cells stained; $+; 5-50 \%$ of neoplastic cells stained; and $\#$; over $50 \%$ of neoplastic cells stained). 


\section{Results}

\section{Normal tissue}

In the normal ductal epithelium, MUC1 gene product detected by both DF3 and $139 \mathrm{H} 2$ was expressed along the luminal surface of the ductal epithelium, while MUC2 gene product detected by anti-MRP and CCP58 was not expressed.

\section{Cancer tissue}

Table 1 shows a summary of the staining results in MCs and IDCs. Fig. 1 shows a summary of the expression patterns and staining densities of MUC1 gene product and MUC2 gene product in each case studied.

\section{Pure mucinous carcinoma}

Most of the pure MCs showed a pattern of positive expression of MUC1 gene product (DF3: 11/11, 139H2: 11/11) and positive expression of MUC2 gene product (anti-MRP: 10/11, CCP58: 11/11) (Fig. 2).

\section{Mixed mucinous carcinoma}

In most of the mixed MCs, the MC areas showed a pattern of positive expression of MUC1 gene product (DF3: 6/6, 139H2: 6/6) and positive expression of MUC2 gene product (anti-MRP: 5/6, CCP58: 6/6), whereas the IDC areas showed a pattern of positive expression of MUCl gene product (DF3: 6/6, 139H2: 6/6) and negative expression of MUC2 gene product (anti-MRP: 0/6, CCP58: 0/6) (Figs. 3, 4).

\section{Invasive ductal carcinoma}

Most of the IDCs showed a pattern of positive expression of MUC1 gene product (DF3: 54/57, 139H2: 53/57) and negative expression of MUC2 gene product (antiMRP: 0/57, CCP58: 0/57), but never showed a pattern of positive expression of both MUC1 and MUC2 gene products.

\section{Expression patterns of MUC1 and MUC2 gene products in cancer tissues}

MUC1 gene product detected by both DF3 and $139 \mathrm{H} 2$ was expressed mainly at the cell membrane, and also in the

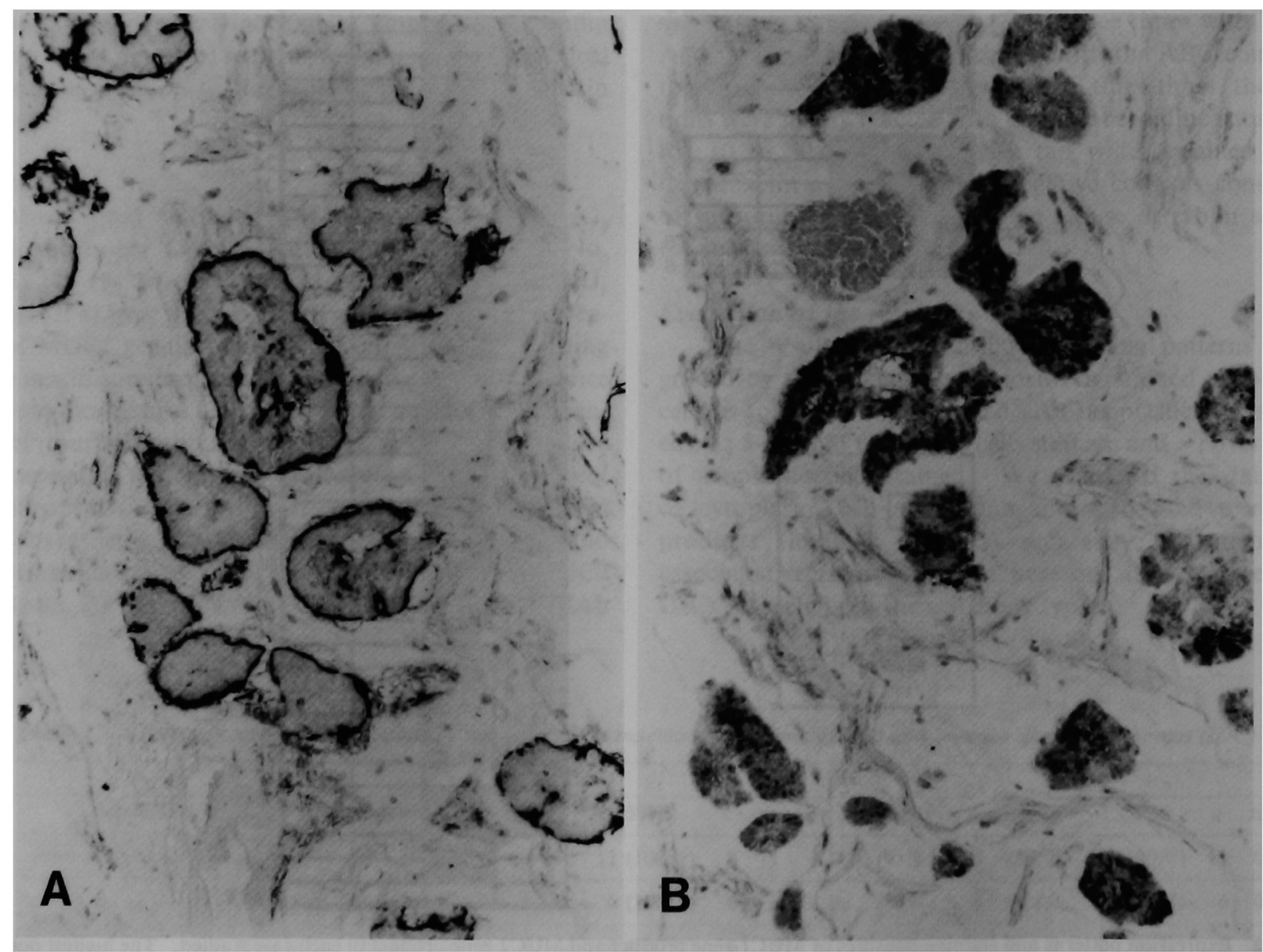

Fig. 2. Immunohistochemical staining pattern of the pure MC. The specimen showed positive expression of $\mathrm{MUC1}$ gene product detected by DF3 mainly at the cell membrane (A) and positive expression of MUC2 gene product detected by anti-MRP exclusively in the cytoplasm (B). $\times 350$. 
cytoplasm and in the associated secretory products, in the pure MCs (Fig. 2A), in both MC areas (Fig. 4A) and IDC areas (Fig. 4C) of the mixed MCs, and in the IDCs (Table 1).

On the other hand, MUC2 gene product detected by anti-MRP and CCP58 was expressed exclusively in the cytoplasm, in the pure MCs (Fig. 2B) and in MC areas (Fig. 4B) of the mixed MCs, whereas never expressed in IDC areas (Fig. 4D) and in the IDCs (Table 1).

\section{Discussion}

As expected, MCs of the breast expressed MUC2 gene product in their cytoplasm, in addition to the expression of MUC1 gene product. MUC1 mucin contains transmembrane domains and are synthesized as membrane protein rather than as secreted proteins [4]. In contrast, MUC2 glycoprotein is a secretory mucin having no transmembrane domains, according to the working model of the MUC2 glycoprotein proposed by Toribara et al. [29] and

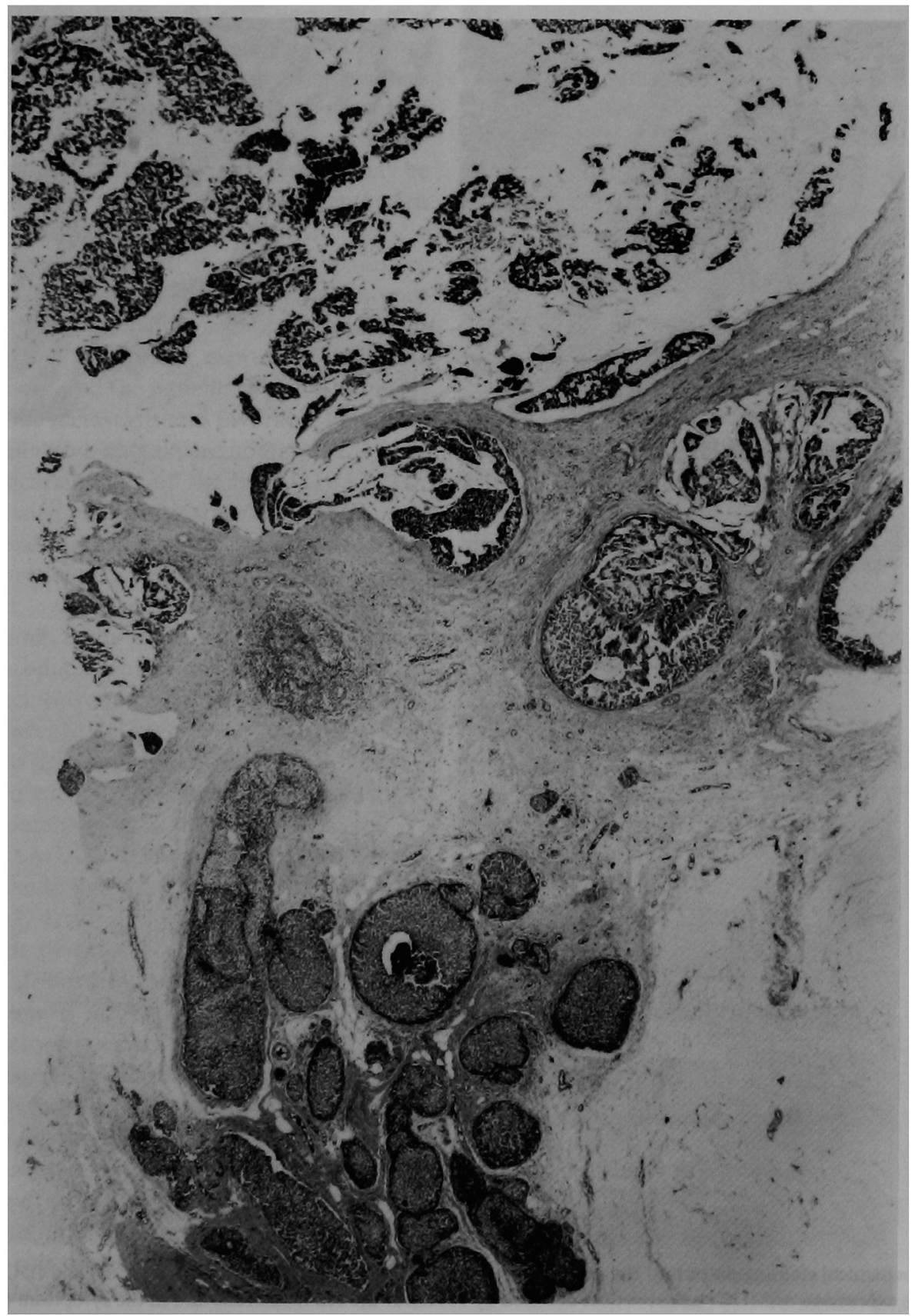

Fig. 3. Hematoxylin and eosin staining of the mixed MC comprising MC area (upper side) and IDC area (lower side). The expression of MUC1 gene product and MUC2 is shown in Fig. 4. $\times 50$. 


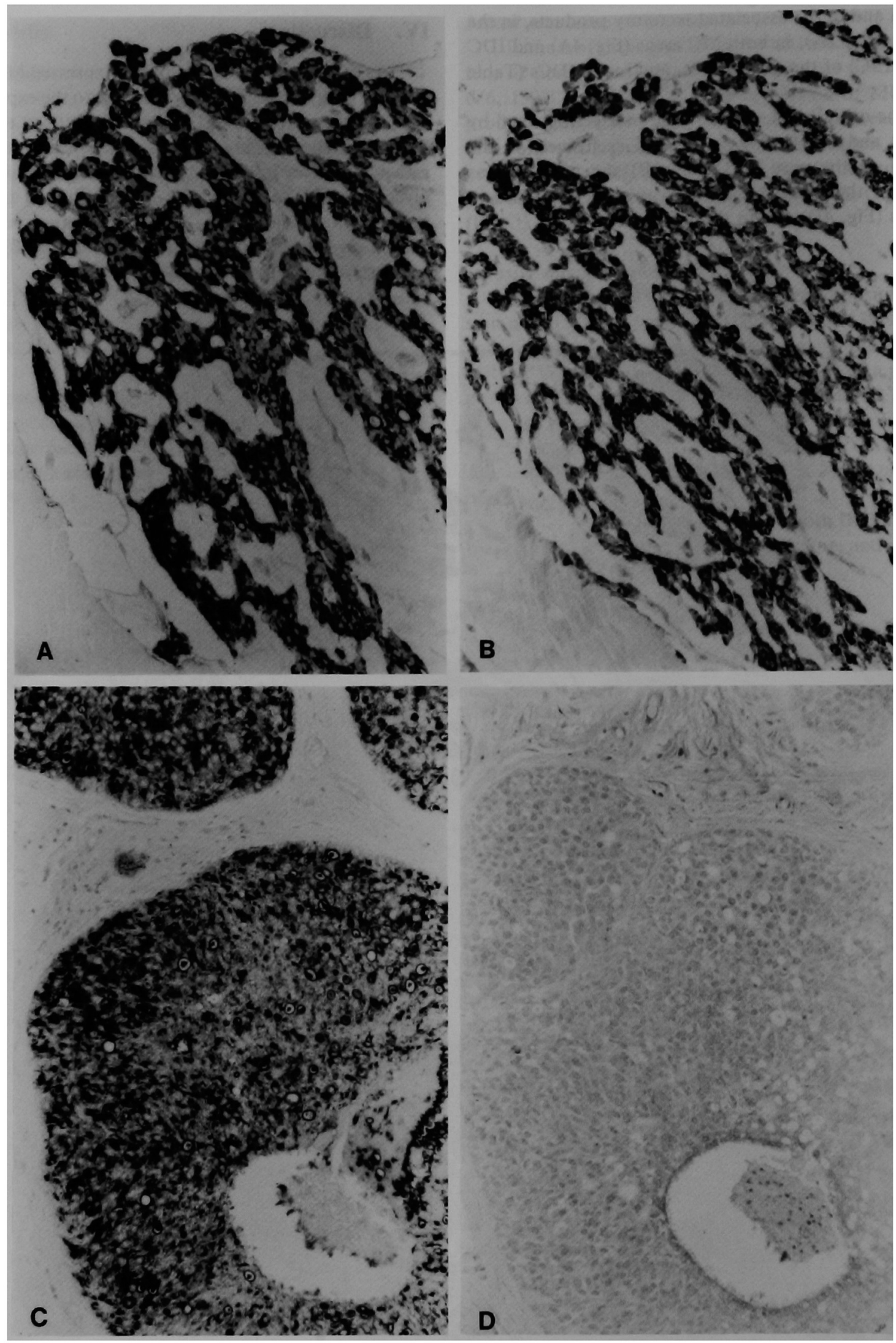

Fig. 4. Immunohistochemical staining pattern of the mixed MC (shown in Fig. 3) comprising MC area (A, B) and IDC area (C, D). The MC area showed positive expression of $\mathrm{MUCl}$ gene product detected by $139 \mathrm{H} 2$ at the cell membrane and in the cytoplasm (A) and positive expression of MUC2 gene product detected by CCP58 exclusively in the cytoplasm (B). The IDC area showed positive expression of MUC1 gene product detected by $139 \mathrm{H} 2$ at the cell membrane and in the cytoplasm (C) but negative expression of MUC2 gene product detected by CCP58 (D). $\times 300$. 
by Gum et al [5]. They described that the presence of multiple cysteine residues in the MUC2 glycoprotein strongly supports the idea that MUC2 codes for a secreted mucin, because disulfide bonding is necessary for the formation of a mucus gel. In MCs showing expression of both MUC1 and MUC2 gene products, MUC1 may encode the membrane protein, and MUC2 may encode the secreted mucin which is abundant around the carcinoma cells. Namely, MUC2 gene expression is strongly related with the formation of the special histologic configuration in MC because of the production of abundant extracellular mucin. Recently, we have succeeded in in situ hybridization that showed MUC2 messenger RNA in MCs (unpublished data). Komaki et al. [14] suggested that floating cancer cells in abundant mucus without having contact with the stroma is important for a favorable prognosis in MC patients. They also commented that abundant mucus within the tumor acts as a barrier to cancerous extension in MC. Hence, the MUC2 gene expression may be related with the favorable prognosis of the patients with MC through the production of abundant mucin.

In our previous studies of intraductal papillary tumors of the pancreas and bile-duct cystadenocarcinomas of the liver, both tumors usually produce an abundant amount of extracellular mucin and express MUC2 gene product predominantly, and the patients with both tumors show little lymph node metastasis and favorable outcomes compared with the invasive ductal carcinomas of the pancreas and cholangiocarcinomas of the liver $[21,32,33]$. The finding of MC with plentiful extracellular mucin and dominant MUC2 gene product expression were analogous to the finding of intraductal papillary tumors of the pancreas and bile-duct cystadenocarcinomas of the liver. However, our previous study of ovarian tumors showed that MUC2 gene product expression increased with the transition of the mucinous tumors from a benign to malignant state [28]. Hence, MUC2 gene product expression is not always related to a favorable prognosis.

Mucin glycoproteins are heavily glycosylated structures, and approximately $80 \%$ of the mass of mucins are carbohydrates [18]. MUC2 gene product expression was usually intracytoplasmic but was not seen in the secreted mucin in MC (Figs. 2, 4) nor in the other tumors described above. The pattern of the intracytoplasmic expression may indicate the following possibilities: (1) MUC2 polypeptide back bone is not heavily glycosylated in the cytoplasm and therefore is easily detected by antibodies; (2) Heavily glycosylated MUC2 gene product is present in secreted mucin, and is hardly detected by antibodies due to blocking of binding of the antibodies to the polypeptide back bone by the carbohydrate chains. Further investigation of the carbohydrates linked to MUC2 polypeptide back bone in the secreted mucin may be important to know the biological role of the extracellular mucin in MC.

Rasmussen et al. [23] reported that the pure and mixed MCs differed siginificantly with respect to a number of prognostic factors, the most important of which was lymph node status. They also described that patients with pure MC had significantly less lymph node metastasis at the time of primary operation and a significantly longer recurrence-free survival than patients with mixed MC. The present study indicated that IDC areas of the mixed MCs never expressed MUC2 gene product, but expressed only MUC1 gene product as shown in Fig. 4. These areas have the same property of mucin production as the pure IDCs have [19], and may be related with poor prognosis of the patients with mixed MC.

\section{Acknowledgments}

The authors thank Dr. H. Yoshinaka (First Department of Surgery, Kagoshima University School of Medicine) and Dr. M. Tokunaga (Department of Pathology, Kagoshima Municipal Hospital) for their kind offer of clinical materials and information, and also thank Mr. Y. Atsuji and Ms. Y. Arimura for their technical assistance.

\section{References}

1. Abe, M. and Kufe, D.: Characterization of cis-acting elements regulating transcription of the human DF3 breast carcinomaassociated antigen (MUC1) gene. Proc. Natl. Acad. Sci. USA 90; 282-286, 1993.

2. Clayton, F.: Pure mucinous carcinomas of breast: Morphologic features and prognostic correlates. Hum. Pathol. 17; 34-38, 1986.

3. Gendler, S. J., Lancaster, C. A., Taylor-Papadimitriou, J., Duhig, T., Peat, N., Burchell, J., Pemberton, L., Lalani, E.$\mathrm{N}$. and Wilson, D.: Molecular cloning and expression of human tumor-associated polymorphic epithelial mucin. $J$. Biol. Chem. 265; 15286-15293, 1990.

4. Gum, J. R.: Mucin genes and the proteins they encode: Structure, diversity, and regulation. Am. J. Respir. Cell Mol. Biol. 7; 557-564, 1992.

5. Gum, J. R., Hicks, J. W., Toribara, N. W., Siddiki, B. and Kim, Y.S.: Molecular cloning of human intestinal mucin (MUC2) cDNA: identification of the amino terminus and overall sequence similarity to Pre-Pro-von Willebrand factor. J. Biol. Chem. 269; 2440-2446, 1994.

6. Gum, J. R., Byrd, J.C., Hicks, J.W., Toribara, N. W., Lamport, D. T. A. and Kim, Y.S.: Molecular cloning of human intestinal mucin cDNAs. J. Biol. Chem. 264; 6480$6487,1989$.

7. Hilkens, J. and Buijs, F.: Biosynthesis of MAM-6, an epithelial sialomucin: Evidence for involvement of a rare proteolytic cleavage step in the endoplasmic reticulum. J. Biol. Chem. 263; 4215-4222, 1988.

8. Hilkens, J., Kroezen, V., Buijs, F., Hilgers, J., Van Der Vilet, M., De Voogd, W., Bonfrer, J. and Bruning, P. F.: MAM-6, a carcinoma associated marker: preliminary characterization and detection in sera of breast cancer patients. In "Monoclonal Antibodies and Breast Cancer", ed. by R. L. Ceriani, Martinus Nijhoff, The Hague, The Netherlands, 1985, pp. 28-42.

9. Inoue, M., Ton, S., Ogawa, H. and Tanizawa, O.: Expression of Tn and sialyl-Tn antigen in tumor tissues of ovary. Am.J. Clin. Pathol. 96; 711-716, 1991.

10. Inoue, M., Ogawa, H., Tanizawa, O., Kobayashi, Y., Tsujimoto, M. and Tsujimura, T.: Immunodetection of sialyl-Tn antigen in normal, hyperplastic and cancerous tissues of the 
uterine endometrium. Virchows Archiv. A. Pathol. Anat. 418; $157-162,1991$.

11. Itzkowitz, S. H., Yuan, M., Montgomery, C. K., Kjeldsen, T., Takahashi, H. K., Bigbee, W. L. and Kim, Y.S.: Expression of $\mathrm{Tn}$, Sialosyl-Tn, and $\mathrm{T}$ antigens in human colon cancer. Cancer Res. 49; 197-204, 1989.

12. Kim, Y.S. and Byrd, J. C.: Colonic and pancreatic mucin glycoproteins expressed in neoplasia. In "Biochemical and Molecular Aspect of Selected Cancers," vol. 1, ed. by T. G. Pretlow II and T. P. Pretlow, Academic Press, New York, 1991, pp. 277-311.

13. Kjeldsen, T., Clausen, H., Hirohashi, S., Ogawa, T., Iijima, H. and Hakomori, S.: Preparation and characterization of monoclonal antibodies directed to the tumor-associated O-linked Sialosyl-2-6 $\alpha-\mathrm{N}$-acetylgalactosaminyl (Sialosyl-Tn) epitope. Cancer Res. 48; 2214-2220, 1988.

14. Komaki, K., Sakamoto, G., Sugano, H., Morimoto, T. and Monden, Y.: Mucinous carcinoma of the breast in Japan: A prognostic analysis based on morphologic features. Cancer 61; 989-996, 1988.

15. Kufe, D., Inghirami, G., Abe, M., Hayes, D., Justi-Wheeler, H. and Schlom, J.: Differential reactivity of a novel monoclonal antibody (DF3) with human malignant versus benign breast tumors. Hybridoma 3; 223-232, 1984.

16. Lan, M. S., Batra, S. K., Qi, W., Metzger, R. S. and Hollingsworth, M.A.: Cloning and sequencing of a human pancreatic mucin cDNA. J. Biol. Chem. 265; 15294-15299, 1990.

17. Ligtenberg, M. J. L., Vos, H. L., Gennisen, A. M. C. and Hilkens, J.: Episialin, a carcinoma-associated mucin, is generated by a polymorphic gene encoding splice variants with alternative amino termini. J. Biol. Chem. 265; 5573-5578, 1990.

18. Neutra, M. R. and Forstner, J.F.: Gastrointestinal mucus: Synthesis, secretion, and function. In "Physiology of the Gastrointestinal Tract", ed. by L. R. Johnson, Raven Press, New York, 1987, pp. 975-1009.

19. Nomoto, M., Yonezawa, S., Tokunaga, M., Kim, Y.S. and Sato, E.: Mucin antigens expression and $\mathrm{Ki}-67$ labeling in breast cancer: The peculiarity in scirrhous carcinoma. Pathol. Int. 45; 233-239, 1995.

20. Norris, H. J. and Tayler, H. B.: Prognosis of mucinous (gelatinous) carcinoma of the breast. Cancer 18; 879-885, 1965.

21. Osako, M., Yonezawa, S., Siddiki, B., Huang, J., Ho, J. J. L., Kim, Y. S. and Sato, E.: Immunohistochemical study of mucin carbohydrates and core proteins in human pancreatic tumors. Cancer 71; 2191-2199, 1993.

22. Peat, N., Gendler, S. J., Lalani, E. N., Duhig, T. and TaylorPapadimitriou, J.: Tissue-specific expression of a human polymorphic epithelial mucin (MUCl) in transgenic mice. Cancer Res. 52; 1954-1960, 1992.

23. Rasmussen, B. B., Rose, C. and Christensen, I.: Prognostic factors in primary mucinous carcinoma. Am. J. Clin. Pathol. 87 ; 155-160, 1987.

24. Sekine, H., Ohno, T. and Kufe, D. W.: Purification and characterization of a high molecular weight glycoprotein detectable in human milk and breast carcinomas. J. Immunol. 135; 3610-3615, 1985

25. Siddiqui, J., Abe, M., Hayes, D., Shani, E., Yunis, E. and Kufe, D.: Isolation and sequencing of a cDNA coding for the human DF3 breast carcinoma-associated antigen. Proc. Natl. Acad. Sci. USA 85; 2320-2323, 1988.

26. Springer, G. F.: $\mathrm{T}$ and $\mathrm{Tn}$, General carcinoma autoantigens. Science 224; 1198-1206, 1984.

27. Takahashi, H.K., Metoki, R. and Hakomori, S.: Immunoglobulin G3 monoclonal antibody directed to $\mathrm{Tn}$ antigen (tumor-associated $\alpha-\mathrm{N}$-acetylgalactosaminyl epitope) that does not cross-react with Blood Group A Antigen. Cancer Res. 48; 4361-4367, 1988.

28. Tashiro, Y., Yonezawa, S., Kim, Y.S. and Sato, E.: Immunohistochemical study of mucin carbohydrates and core proteins in human ovarian tumors. Hum. Pathol. 25; 364-372, 1994.

29. Toribara, N. W., Gum, J. R., Culhane, P. J., Lagace, R. E., Hicks, J. W., Petersen, G. M. and Kim, Y. S.: MUC-2 human small intestinal mucin gene structure: Repeated arrays and polymorphism. J. Clin. Invest. 88; 1005-1013, 1991.

30. Wreschner, D. H., Hareuveni, M., Tsarfaty, I., Smorodinsky, N., Horev, J., Zaretsky, J., Kotkes, P., Weiss, M., Lathe, R., Dion, A. and Keydar, I.: Human epithelial tumor antigen cDNA sequences. Differential splicing may generate multiple protein forms. Eur. J. Biochem. 189; 463-473, 1990.

31. Xing, P.-X., Prenzoska, J., Layton, G. T., Devine, P. L. and McKenzie, I. F. C.: Second generation monoclonal antibodies to intestinl MUC2 peptide reactive with colon cancer. J. Natl. Cancer Inst. 84; 699-703, 1992.

32. Yamashita, K., Yonezawa, S., Tanaka, S., Shirahama, H. Sakoda, K., Imai, K., Xing, P.-X., McKenzie, I. F. C., Hilkens, J., Kim, Y.S. and Sato, E.: Immunohistochemical study of mucin carbohydrates and core proteins in hepatolithiasis and cholangiocarcinoma. Int. J. Cancer 55; 8291, 1993.

33. Yonezawa, S.: Application of immunohistochemistry for diagnosis of neoplasms: Mucin antigens expression and biological behavior of neoplasms. Acta Histochem. Cytochem. 27; 561-566, 1994.

34. Yonezawa, S., Byrd, J. C., Dahiya, R., Ho, J. J. L., Gum, J. R., Griffiths, B., Swallow, D. M. and Kim, Y. S.: Differential mucin gene expression in human pancreatic and colon cancer cells. Biochem. J. 276; 599-605, 1991.

35. Yonezawa, S., Tachikawa, T., Shin, S. and Sato, E.: SialosylTn antigen: Its distribution in normal human tissues and expression in adenocarcinomas. Am. J. Clin. Pathol. 98; 167-174, 1992. 